ARTICLE

Received 14 Feb 2016 | Accepted 29 Jun 2016 | Published 4 Aug 2016

DOI: $10.1038 /$ ncomms12401

OPEN

\title{
Mass production of shaped particles through vortex ring freezing
}

Duo An¹, Alex Warning ${ }^{1}$, Kenneth G. Yancey ${ }^{1}$, Chun-Ti Chang ${ }^{2}$, Vanessa R. Kern ${ }^{2}$, Ashim K. Datta ${ }^{1}$, Paul H. Steen ${ }^{2}$, Dan Luo ${ }^{1,3,4}$ \& Minglin Ma ${ }^{1}$

A vortex ring is a torus-shaped fluidic vortex. During its formation, the fluid experiences a rich variety of intriguing geometrical intermediates from spherical to toroidal. Here we show that these constantly changing intermediates can be 'frozen' at controlled time points into particles with various unusual and unprecedented shapes. These novel vortex ring-derived particles, are mass-produced by employing a simple and inexpensive electrospraying technique, with their sizes well controlled from hundreds of microns to millimetres. Guided further by theoretical analyses and a laminar multiphase fluid flow simulation, we show that this freezing approach is applicable to a broad range of materials from organic polysaccharides to inorganic nanoparticles. We demonstrate the unique advantages of these vortex ring-derived particles in several applications including cell encapsulation, three-dimensional cell culture, and cell-free protein production. Moreover, compartmentalization and ordered-structures composed of these novel particles are all achieved, creating opportunities to engineer more sophisticated hierarchical materials.

\footnotetext{
${ }^{1}$ Department of Biological and Environmental Engineering, Cornell University, Ithaca, New York 14853, USA. ${ }^{2}$ School of Chemical and Biomolecular Engineering, Cornell University, Ithaca, New York 14853, USA. ${ }^{3}$ Kavli Institute at Cornell for Nanoscale Science, Cornell University, Ithaca, New York 14853 , USA. ${ }^{4}$ Suzhou Institute of Nano-Tech and Nano-Bionics, Chinese Academy of Sciences, Suzhou 215123, China. Correspondence and requests for materials should be addressed to D.L. (email: dan.luo@cornell.edu) or to M.M. (email: mm826@cornell.edu).
} 
vortex ring is a torus-shaped, fluidic region where the fluid spins around an imaginary axis line. Vortex rings exist almost ubiquitously in nature ${ }^{1-4}$ and have stimulated numerous studies for decades ranging from classical fluid mechanics ${ }^{5-14}$ to aquatic propulsion ${ }^{15,16}$, cardiac flows ${ }^{17-19}$, hydropropulsion/aeropropulsion 20 , micro jet thrusters ${ }^{21}$, and multi-scale stirring and mixing ${ }^{22}$. One simple way to generate a vortex ring is to have a droplet impacting the surface of a miscible liquid. When hitting the surface, the nearly spherical droplet deforms; during this deformation, many fluid intermediates with various intriguing, non-spherical shapes appear, including those resembling teardrops, jellyfishes, caps and donuts.

However, vortex rings rapidly evolve and are often shortlived $^{23}$, making it almost impossible to harvest and utilize them as materials. In search of a novel material that can generate various, non-spherical shapes, we discover that a crosslinkable nanoclay system can be controlled to freeze vortex rings, creating a new class of vortex ring-derived particles (VRP) with uniform and sometimes unprecedented shapes including the teardrop-, jellyfish-, cap- and donut-shaped ones. Further adoption of a simple and inexpensive electrospraying technique enables the mass production of the VRP with controllable dimensions ranging from millimetres down to hundreds of microns (that is, microVRP). More importantly, we further show that it is possible to fabricate the microVRP from almost any materials as long as there exists a proper 'freezing event', where the unstable liquid vortex rings are fixed into stable hydrogel or solid microparticles of a defined shape through a gelation or precipitation process. Theoretical analyses and a multiphase laminar fluid flow simulation also show that this freezing method, regardless what materials are used, can be a universal fabrication platform to make microVRP. Indeed, besides nanoclay, we are able to produce microVRP from several other very different materials, from polysaccharides (for example, alginate, chitosan) to silica nanocolloids, all by proper designs and control of the vortex ring freezing. Similarly, we can also fabricate hybrid microVRP from various material mixtures with functional components such as magnetic nanoparticles (NPs).

Among the different shapes of the microVRP, the donut (or toroidal) one is of special interests in the materials science field ${ }^{24-26}$. Compared with the more common spherical shapes, donut ones have several prominent advantages including always a higher surface/volume ratio, a shorter diffusion path within, and a better deformability. These advantages make the donutshaped particles highly desirable in many applications such as catalytic reactions ${ }^{27}$, cell encapsulation ${ }^{24}$ and structural materials construction ${ }^{28}$. Several applications of these donutmicroVRP are demonstrated including bioencapsulation, threedimensional (3D) cell culture, and cell-free protein production. In addition, we show the successful fabrication of more complicated Janus and core-shell donut-microVRP by engineering the electrospray nozzle. The donut-microVRP can also be directionally and orderly organized in either linear or planar fashion by taking advantage of their unique geometry, paving the way for future assembly of more sophisticated hierarchical materials.

\section{Results}

Fabrication of VRP by freezing vortex rings. A simple way to form a vortex ring is to drop a droplet into a miscible liquid ${ }^{9,28}$. In a typical process, when a droplet hits the free surface of a miscible liquid at a sufficient impact speed, the droplet starts to deform in order to dissipate the energy by curling back (see Fig. 1a for the time sequence). As the edge continues to curl, the centre of the droplet becomes thinner and thinner.
Eventually, when the centre is too thin to withstand the surface tension, it breaks and a donut shape is formed (Fig. 1b).

To more precisely visualize the vortex ring formation and investigate whether it is possible to utilize this process to fabricate novel particles, we need a material that allows controllable 'freezing' of the vortex ring (Supplementary Note 1). We discover that a nanoclay system is ideal here because of its unique, controllable gelation properties. The nanoclay (Laponite XLG) possess a disk-like structure with a non-homogeneous charge distribution: negative charges on the disk surface and positive charges on the rim $^{29}$. This special structure endows nanoclay with unique properties: in deionized water, the nanoclay disks are homogeneously distributed; whereas, in the presence of certain ionic species, the nanoclay disks pack together to generate a 'house-of-cards' structure, forming a hydrogel ${ }^{30}$ (Supplementary Fig. 1a). Importantly, the viscosity $(\sim 1.7 \mathrm{cP})$ and surface tension $\left(\sim 72 \mathrm{mN} \mathrm{m}^{-1}\right)$ of the nanoclay solution are well suitable for the formation of vortex rings within the crosslinking buffer, while the gelation speed can be precisely controlled. We used a high-speed camera to capture a series of images of vortex rings formed by a drop of nanoclay solution impacting an aqueous bath, with or without gelation reaction (Fig. 1c). In case of no gelation (that is, in a deionized water), the droplet moves along while spreading out with the front edges curling back. The shape of the droplet is constantly changing, experiencing in order, a teardrop-, jellyfish-, cap- and donut-shape. This whole process lasted for merely $100 \mathrm{~ms}$, and the nanoclay solution finally dispersed homogeneously (see the upper row of Fig. 1c). In contrast, in the presence of gelation (that is, in a $100 \mathrm{mM} \mathrm{CaCl}_{2}$ solution), the vortex ring maintains its shape (that is, formed a VRP) starting from $36 \mathrm{~ms}$ (see the bottom row of Fig. 1c). As a result, by tuning the viscosity and impact speed of the droplet, we successfully captured the intermediates and produced VRP with various intriguing shapes (Fig. 1d,e). Some of these VRP may be difficult to fabricate using other traditional methods such as soft lithography 27,31 , microfluidics ${ }^{32}$ or self-assembly ${ }^{33}$. The formation of each of the VRP was also captured in situ and in real time by the high-speed camera images (Supplementary Fig. 2). Moreover, their unique shapes allow us to assemble the VRP in an ordered fashion, for example, into an orientated, closepacked monolayer via a strategy similar to a Langmuir-Blodgett deposition (Fig. 1f; Supplementary Fig. 3; Supplementary Note 2).

Mass production of microVRP. The aforementioned simple dripping experiment proves the feasibility of freezing vortex rings at different stages to form various VRP. To reduce their dimensions and increase their production rate, we employ an industrially scalable electrospraying technique (Fig. 2a). Briefly, by adding a high voltage electric field between the nozzle and the collector, the liquid jet is charged and breaks up into much smaller droplets ${ }^{34}$. Interestingly, the fluid dynamic behaviour of smaller droplets impacting the crosslinking buffer remains similar to the 'simple dripping' case. As a result, we easily fabricated nanoclay microVRP (for example, with the donut shape) at a very high rate ( $>15,000$ VRP per min) (Fig. 2b). Both the micro-scale torus shape and the nanoscale porous network structure of these donut-microVRP are characterized and confirmed (Supplementary Fig. 1b-d).

Importantly, we discover that the size and shape of the electrosprayed VRP can be well controlled. By simply tuning the voltage, the outer diameter of the donut-microVRP can be controlled, from around $280 \mu \mathrm{m}$ to over $1.5 \mathrm{~mm}$ (Fig. 2c). The microVRP dimensions are also controllable by tuning the working distance between the nozzle and the collecting buffer surface (Supplementary Fig. 4; Supplementary Note 3). 
a

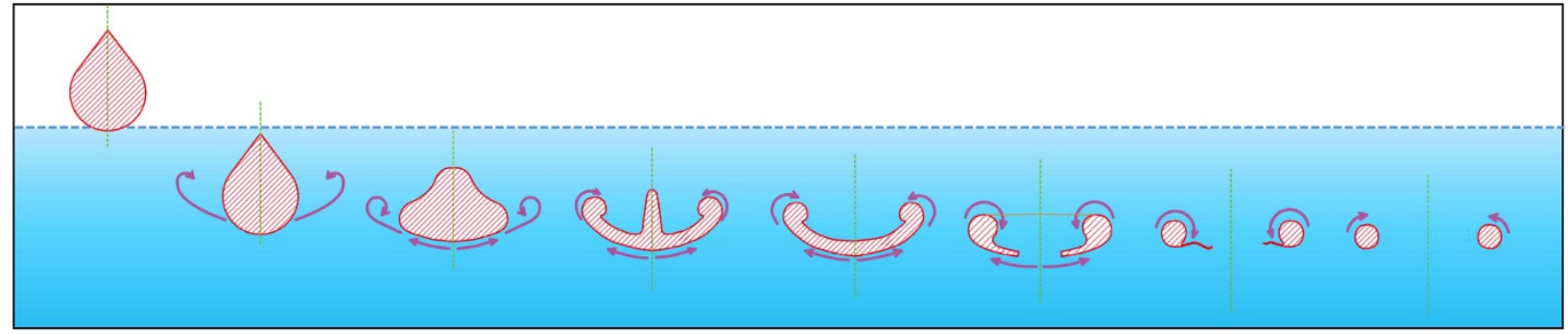

b

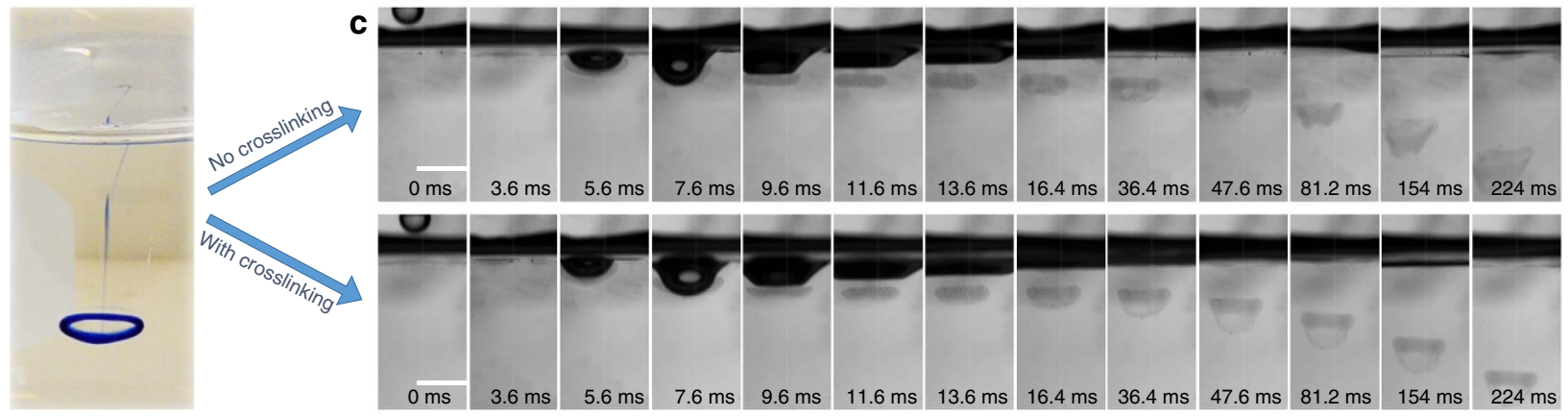

d

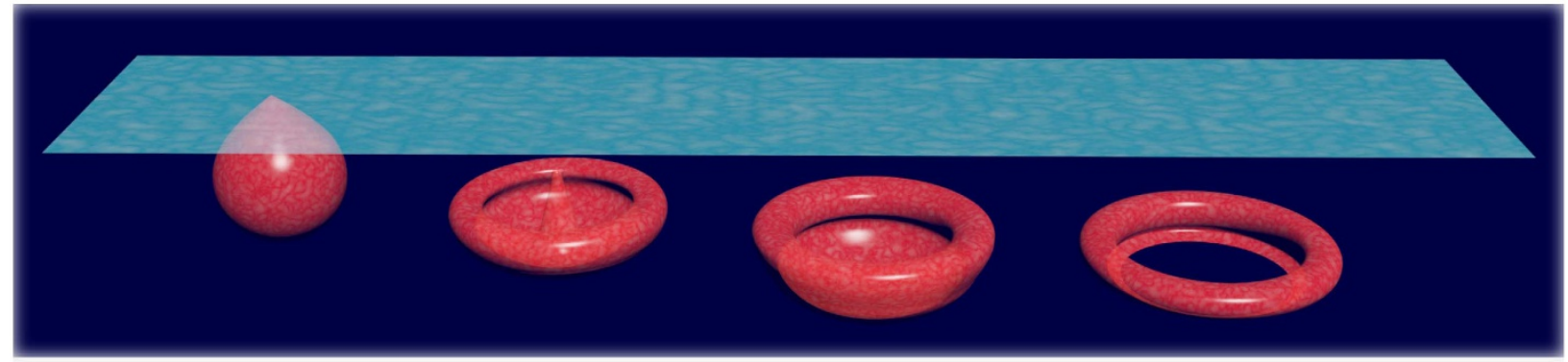

e
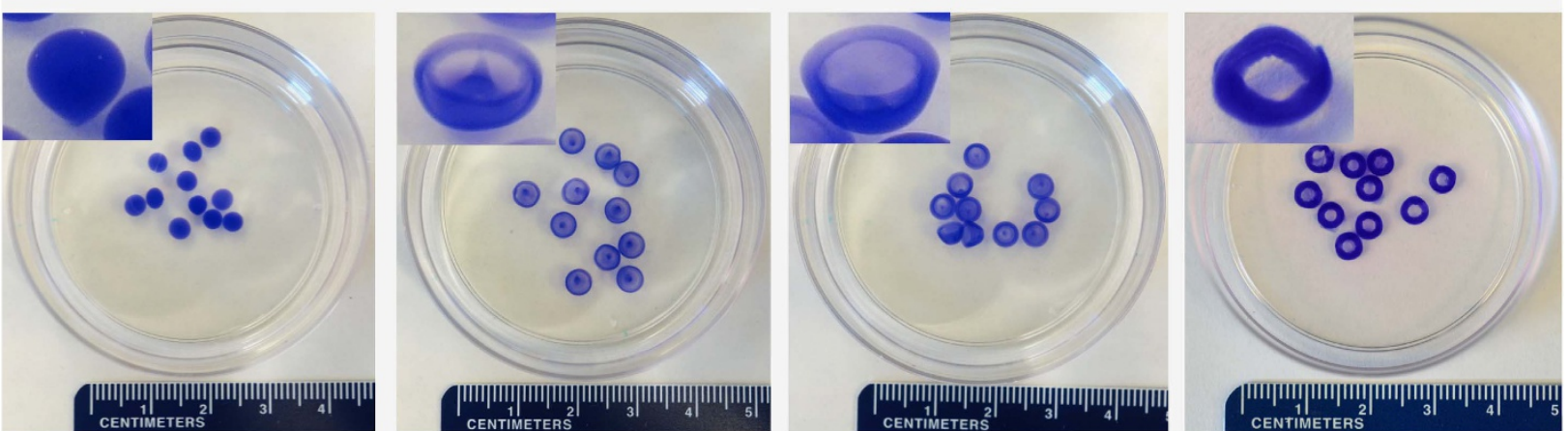

$\mathbf{f}$
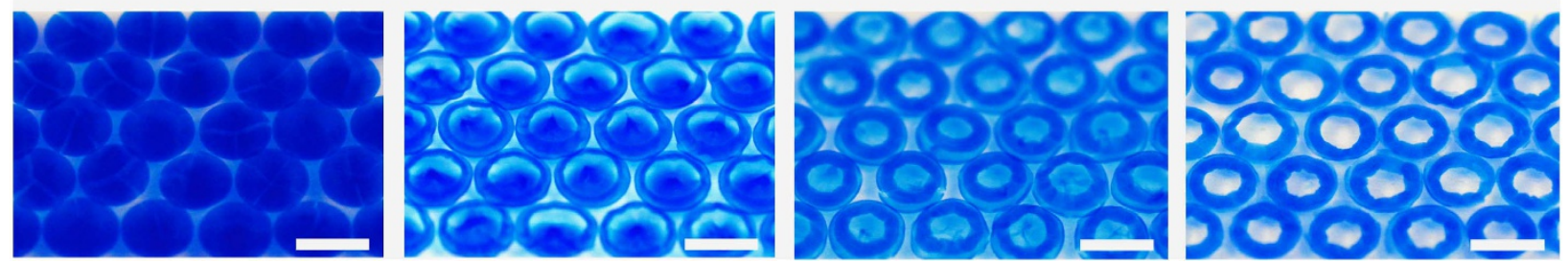

Figure 1 | Droplet-formed vortex ring and VRP made by freezing vortex rings. (a) Schematic illustration of the vortex ring formation process (a water drop striking a water surface). (b) Digital image of a vortex ring formed by dripping a drop of ink into a water pool. (c) High-speed camera images of the vortex ring formation process by dripping a drop of nanoclay solution into a water pool (upper) and into a crosslinking buffer pool (lower). (d-f) Four typical VRP: teardrop-, jellyfish-, cap- and donut-ones. 3D illustration (d), digital images (e) and close-packed monolayers (f). Scale bars, 3 mm (c); 6 mm (f).

Moreover, we can control the shapes of the microVRP by adjusting the viscosity of the nanoclay solution. Similar to the dripping method, we obtained, in addition to the donutmicroVRP, three other types including teardrop-, jellyfish- and cap-shaped ones, all made with high uniformity and in mass numbers (Fig. 2d).
Theoretical analyses and simulation. To better understand the control of size and shape of the nanoclay microVRP, we investigate theoretically how the size of the donut-microVRP or droplets is affected by the processing parameters in electrospraying such as the voltage. A classic scaling law which is valid for liquids with relatively low viscosities and electrical 

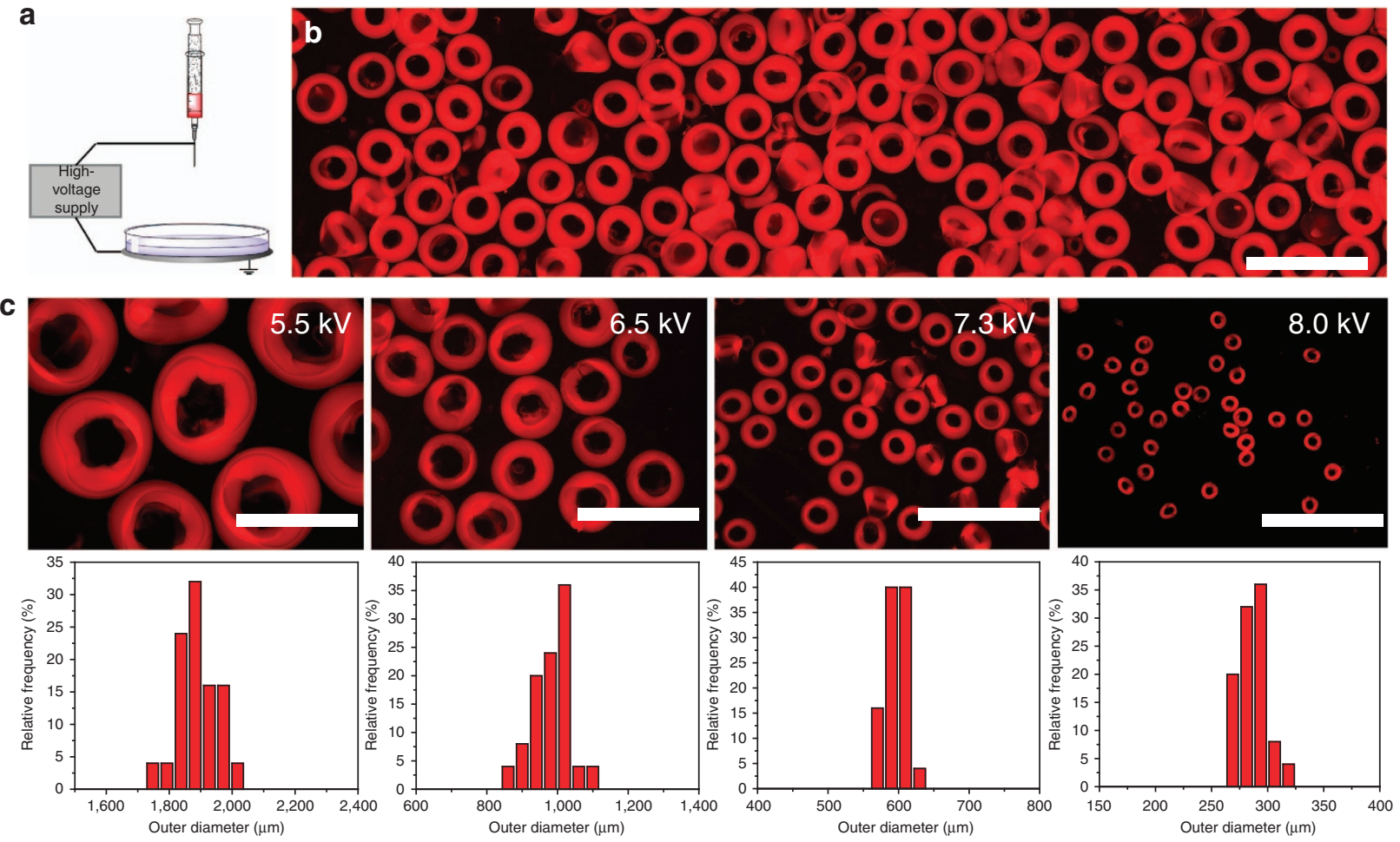

d
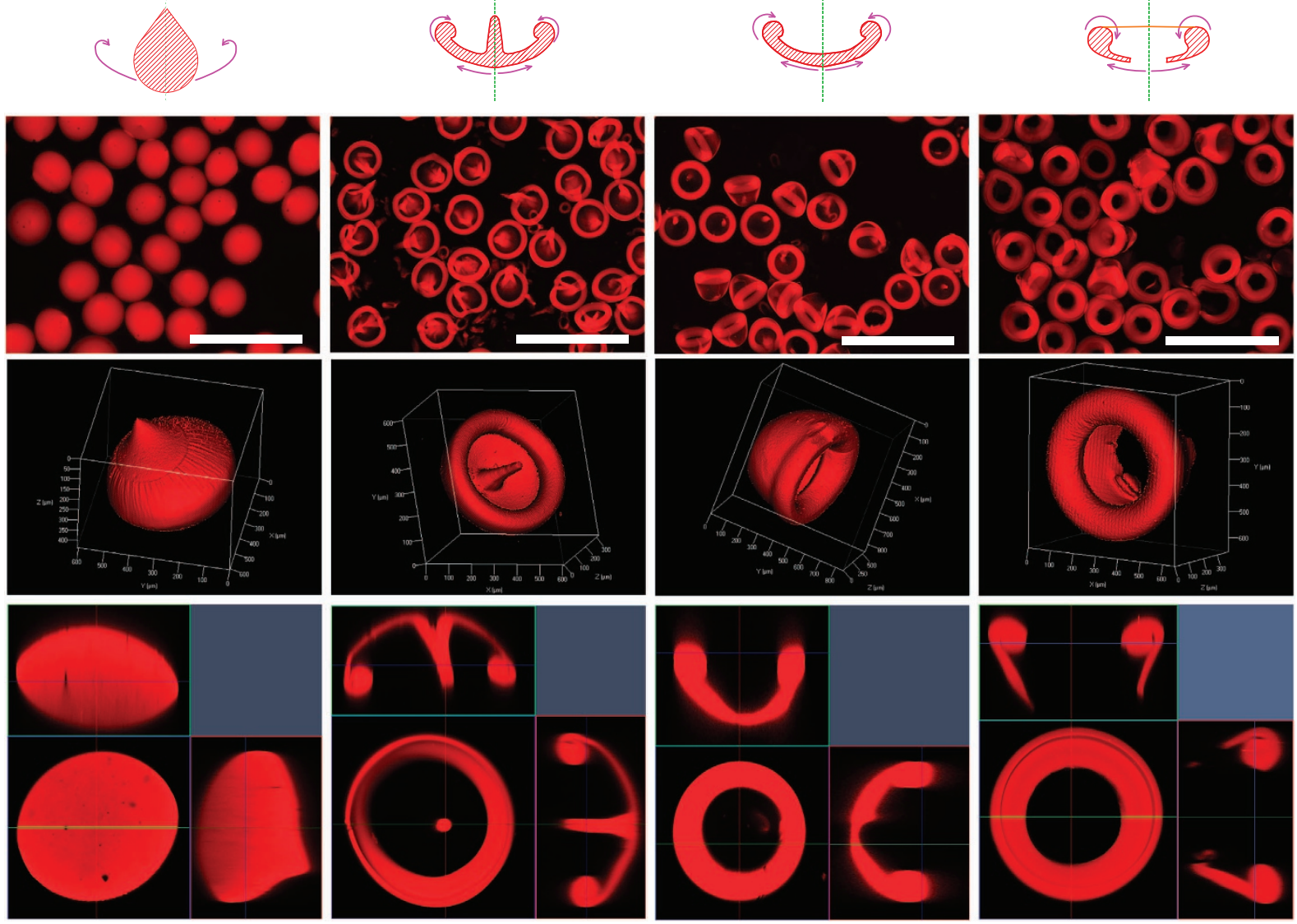

Figure 2 | Mass production and size/shape control. (a) Schematic illustration of the electrospray setup. (b) Microscopic fluorescent images of the nanoclay hydrogel donut-microVRP fabricated by electrosparying. For better visualization, a fluorescent dye Rhodamine B was mixed in the nanoclay solution. (c) Size control of the nanoclay hydrogel donut-microVRP: microscopic images and corresponding size distribution plots. (d) Shape control of the nanoclay hydrogel microVRP by tuning the viscosity of the nanoclay solution and voltage: (from top to bottom) schematic illustrations, microscopic images, 3D confocal microscopic images and cross-sectional images. Scale bars, $2 \mathrm{~mm}(\mathbf{b}) ; 1 \mathrm{~mm}(\mathbf{c}, \mathbf{d})$. 
a

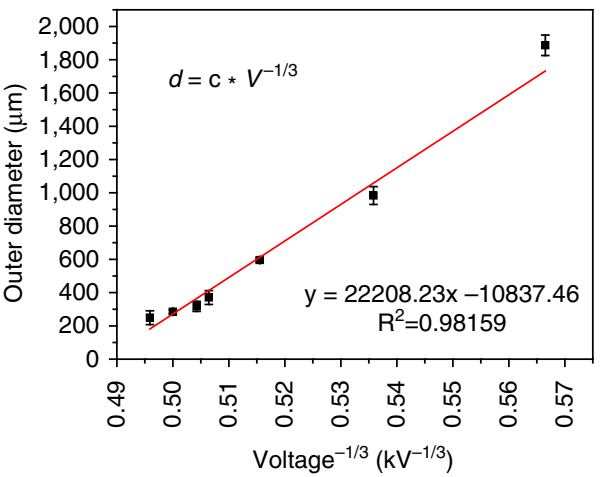

b

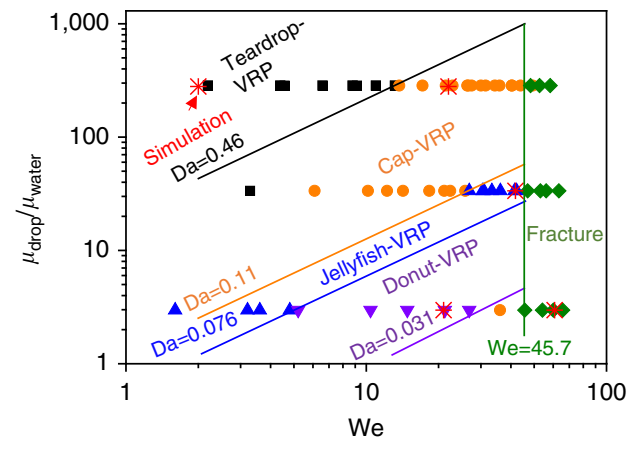

C

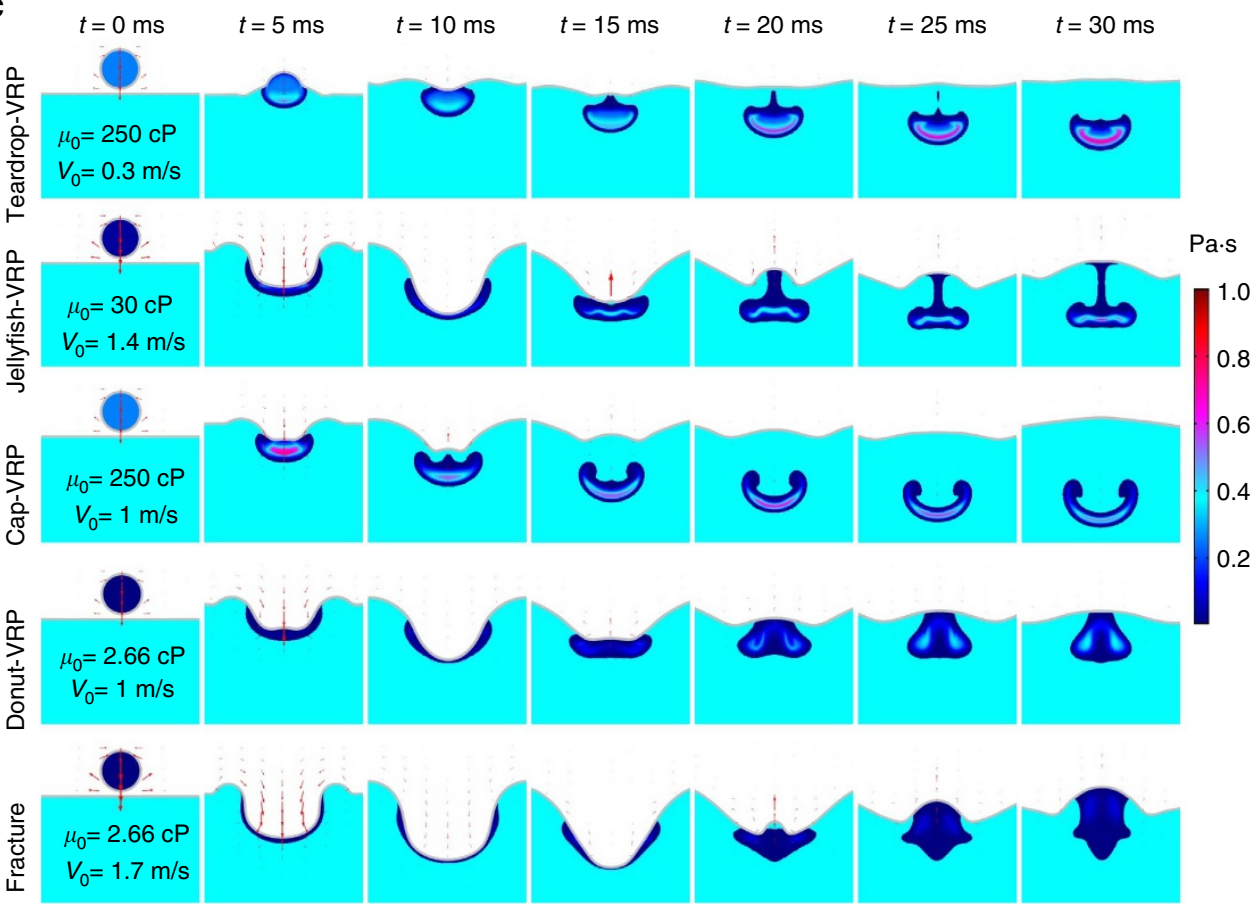

Figure 3 | Mechanistic study and simulation. (a) MicroVRP sizes controlled by tuning voltage. Error bars in a, represent one standard deviation of the data away from the mean. (b) Experimental results and theoretical analysis of the VRP shapes for different parameters. (c) The axisymmetric simulation results of the vortex ring formation over time for five situations. The light blue is the crosslinking bath and the droplet above is the nanoclay solution where the colour represents the viscosity difference. The nanoclay solution is only plotted where there is at least $0.2 \mathrm{wt} \%$ nanoclay particles and the grey line at the air/solution interface represents where there is equal air-water volume fraction.

conductivities indicates the relationship between droplet size $(D)$ and voltage $(V)^{34}$ :

$$
D \sim V^{-1 / 3}
$$

This scaling law suggests that the droplet size will decrease as the voltage increases. As shown in Fig. 3a, experimental data are plotted and fitted in this relationship assuming the droplet size is proportionally related to the VRP size. The fitting result indeed shows a linear relationship with a coefficient of determination $R^{2} \approx 0.9816$.

Next, to understand the controllability of the VRP shape, we conducted a series of experiments and simulations to study how the properties of the drop before impact and after immersion dictated the development of vortex rings (Supplementary Figs 5-8; Supplementary Table 1; Supplementary Note 4). On impact, drop deformation is resisted by interfacial tension while, after immersion, VRP formation depends on the rate of mixing by invasion of vorticity into vorticity-free regions relative to the rate of reaction. Pre-impact ballistics is controlled by Weber (We) and Ohnesorge $(\mathrm{Oh})$ numbers while post-impact vortical invasion and reaction is controlled by Reynolds (Re) and Damköhler (Da) numbers $^{6,8}$ (Supplementary Note 6), defined as

$$
\begin{aligned}
\mathrm{We} & \equiv \frac{\rho v^{2} r}{\sigma} \\
\mathrm{Oh} & \equiv \frac{\mu}{\sqrt{\rho r \sigma}} \\
\mathrm{Re} & \equiv \frac{\rho a \gamma}{\mu} \\
\mathrm{Da} & \equiv \frac{k C}{\gamma}
\end{aligned}
$$

where $\rho$ is the drop's density, $v$ is the impact velocity, $r$ is the radius, $\mu$ is the dynamic viscosity, $\sigma$ is the surface tension, $k$ is the 

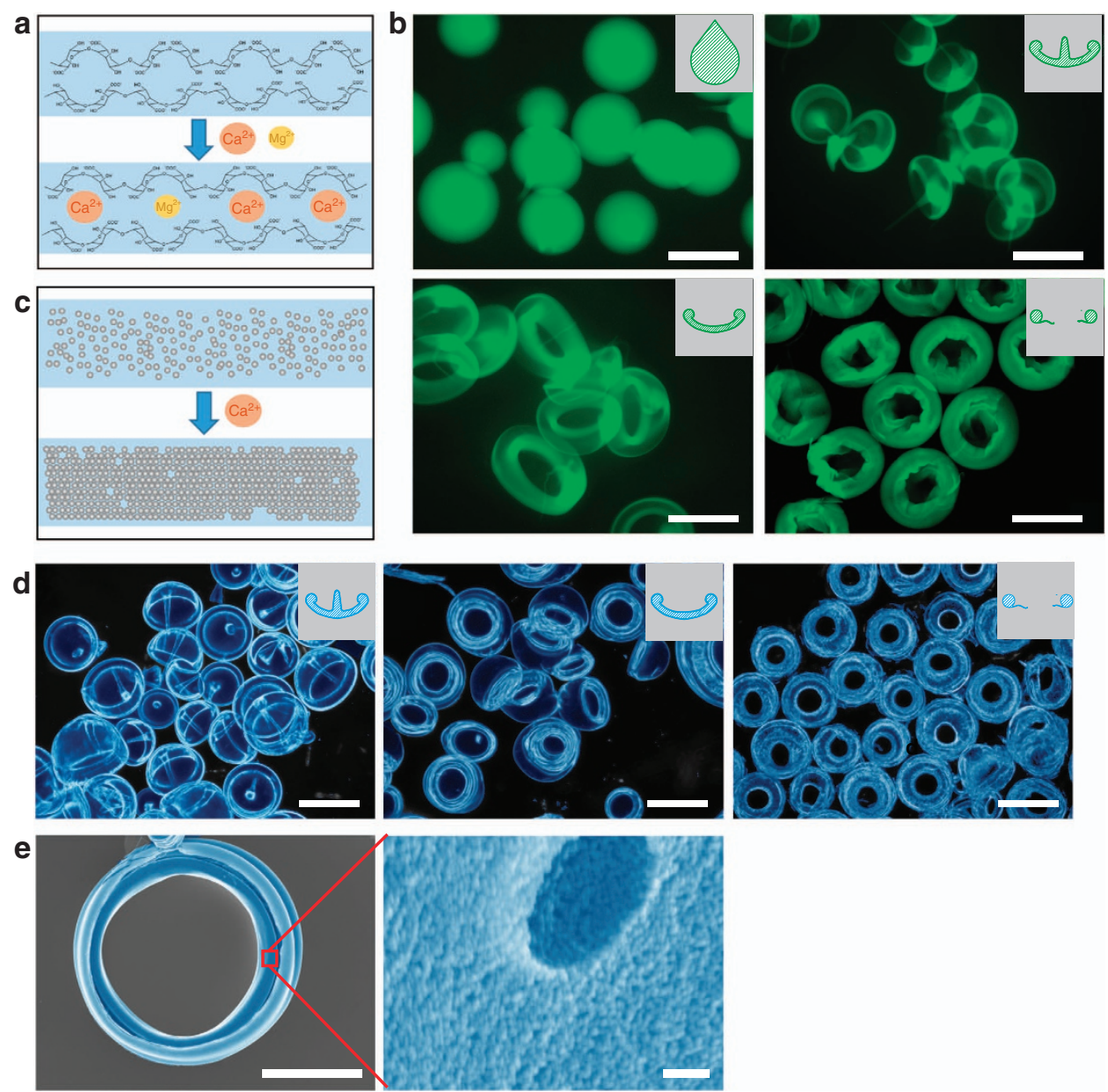

Figure 4 | MicroVRP fabricated from different materials. (a) Scheme of the modified gelation process in the ion-competing crosslinking buffer for alginate. (b) Alginate hydrogel microVRP, alginate labelled with Alexa Fluor 488 dye. (c) Scheme of the silica nanocolloidal precipitation process. (d) Microscopic images of the nanosilica microVRP (false-coloured). (e) SEM images of a dried nanosilica donut-microVRP (false-colored). Scale bars, $500 \mu \mathrm{m}$ (b,d); $60 \mu \mathrm{m}$ (e left panel); $100 \mathrm{~nm}$ (e, right panel).

reaction rate constant, $C$ is the concentration of binding sites, and $a \gamma$ is the rate of formation of interfacial area $a$ between regions with and those without vorticity. Rate $\gamma$ is estimated as a shear rate, from the simulation results. We and $\mathrm{Oh}$ numbers respectively characterize the strength of inertia and viscosity relative to surface tension of the impacting drops. Re and Da numbers respectively characterize rates of vorticity generation $a \gamma$ relative to resistance to invasion of vorticity by viscosity $\mu / \rho$ and rate of crosslinking $k C$ relative to shear rate $\gamma$.

The results from all experiments and simulations are summarized in Fig. 3b,c and Supplementary Information. According to our experimental and simulation results, with low-We, high-Oh and high-Da (that is, reaction dominates mass transport), the droplet is 'frozen' at the early stage of vortex ring development and forms a teardrop VRP. While increasing We or decreasing $\mathrm{Oh}$ in the intermediate $\mathrm{Da}$ range (that is, reaction and mass transport are commensurate), cap- or jellyfish-VRP are obtained. Further increasing We created rotating vortices that punctures the cap's central part to produce a donut-VRP. When the We is large (or Oh low) and the Da sufficiently low (that is, mass transport dominates reaction), the particle cannot maintain its shape as a whole due to the reaction rate being much slower than the transport, the particle ends up fracturing (Supplementary Fig. 9). Normally, unconstrained liquid tori are very unstable and hence difficult to preserve. However, our method points out a simple approach of preserving any shape that a drop may form while impacting the free surface of a crosslinking agent. We, Oh, Re and $\mathrm{Da}$ are the lumped control parameters of solution properties and process. Our work suggests that, regardless what materials are used, an appropriate prescription of the four dimensionless numbers in principle will allow us to obtain a continuously deformed family of all the shapes spanning the developing process of a vortex ring. (Supplementary Note 5)

MicroVRP formed with other materials and reactions. Our modelling and simulation suggests that microVRP can be fabricated from other materials as long as the deformation and 'freezing' of vortex rings can both be controlled. To experimentally test this conclusion, we first chose two popular, crosslinkable biopolymers, alginate and chitosan. These two materials have been processed into hydrogel particles via electrospraying and they have been extensively used for drug and cell deliveries ${ }^{35}$. However, as in almost all previous studies, electrosprayed hydrogel microparticles have been spherical ${ }^{36,37}$. This is as expected according to our mechanistic studies due to the fact that their gelation reactions are relatively fast and that there is no time for the droplets to evolve into vortex rings. For instance, alginate droplet stops changing shape $20 \mathrm{~ms}$ after impacting a 
a

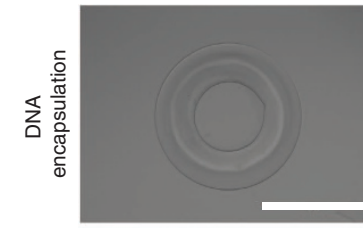

d

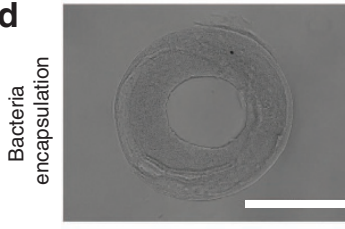

e

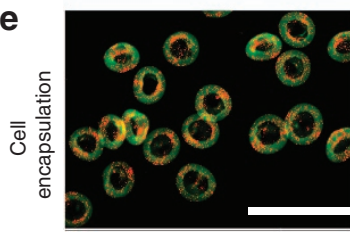

f

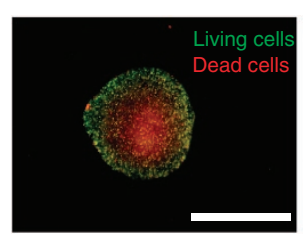

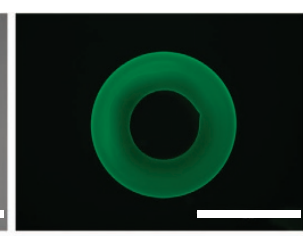
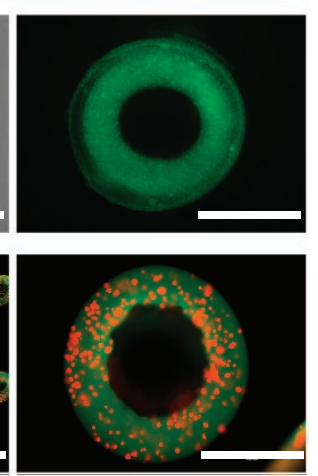

b

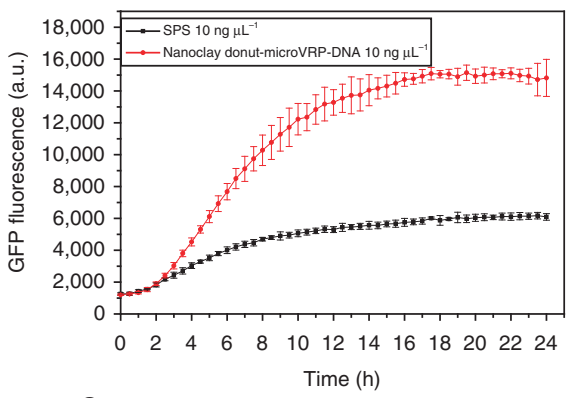

C
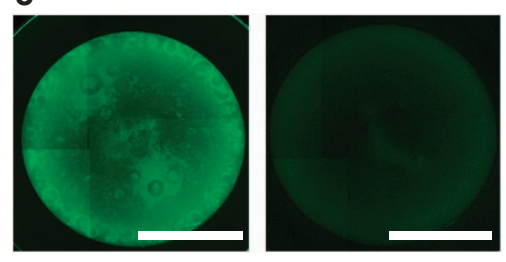

g
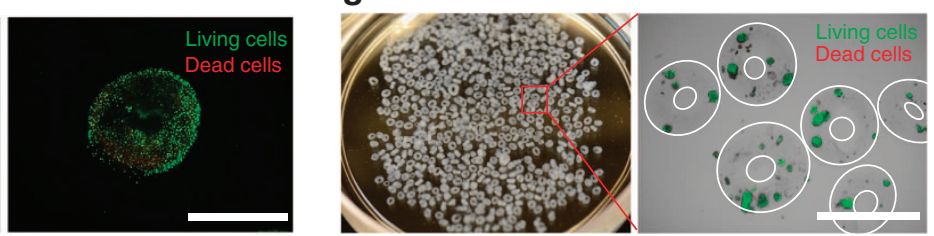

Figure 5 | Bioencapsulation applications using donut-microVRP. (a) A nanoclay hydrogel donut-microVRP encapsulating plasmid DNA: bright-field image and fluorescent image (stained with GelGreen). (b) Kinetic curve of the GFP fluorescence from cell-free protein production. Error bars in $\mathbf{b}$ represent one s.d. of the data away from the mean. (c) Fluorescent images of the reaction wells of cell-free protein production with (left) or without (right) nanoclay donut-microVRP. (d) A nanoclay hydrogel donut-microVRP encapsulating bacteria: bright-field image and fluorescent image (GFP-expressing E. coli). (e) Alginate hydrogel donut-microVRP encapsulating MDA-MB-231 cells that express Tomato red. (f) Cell viability test of the MDA-MB-231 cells encapsulated in an alginate spherical particle and an alginate donut-VRP, 4 days after encapsulation. The cells were stained with calcium-AM (green, live) and ethidium homodimer (red, dead). (g) Rat pancreatic islets encapsulated in the alginate donut-VRP. Scale bars, $400 \mu \mathrm{m}$ (a, d, e right panel); $3 \mathrm{~mm}$ (c); $2 \mathrm{~mm}$ (e left panel, $\mathbf{f}, \mathbf{g}$ ).

conventional crosslinking buffer (Supplementary Fig. 10). Decreasing the crosslinking buffer concentration may slow down the gelation, but the low concentration ion buffers cannot completely crosslink the hydrogel, resulting in fractures (Supplementary Tables 2 and 3; Supplementary Note 7).

Here, to control the freezing event, we developed a novel ioncompeting crosslinking solution containing a primary crosslinking agent (calcium ions for alginate or tripolyphosphate for chitosan) and a competing agent (magnesium ions or dihydrogen phosphate ions). Since the competing agent has a strong resemblance to the primary one but a much weaker crosslinking ability, when the droplet enters the ion-competing crosslinking solution, the competing agent competes with the primary one and hence slows down the reaction (Fig. 4a; Supplementary Fig. 11a). Indeed, using the ion-competing crosslinking solutions we successfully created microVRP from both alginate (Fig. 4b; Supplementary Fig. 12) and chitosan (Supplementary Fig. 11b).

More interestingly, we can fabricate microVRP from inorganic colloidal nanoparticles as well. Silica nanocolloidal solution (ca. $50 \mathrm{~nm}$ in diameter) is initially stabilized in sodium chloride buffer. When the ionic strength is changed, the electrostatic homeostasis among the silica nanoparticles is broken, causing the nanoparticles to aggregate and precipitate (Fig. 4c). This precipitation event is akin to the gelation event. As a result, by electrospraying the silica nanocolloidal solution into an ionic buffer (see Methods for details), we also achieved mass production of uniform nanosilica jellyfish-, cap- and donut-microVRP (Fig. 4d). These microVRP have excellent stability and rigidity; they remain almost intact after air-drying (Fig. 4e). Further energy dispersive spectrometer (EDS) element mapping reveals the uniform distribution of $\mathrm{Si}, \mathrm{O}, \mathrm{Ca}$ and $\mathrm{Cl}$ (Supplementary Fig. 13), confirming the relatively homogeneous precipitation.

Hydorgel donut-microVRP for bioencapsulation. The fact that non-spherical, microVRP can be produced from a wide range of materials makes them not only fundamentally intriguing but also practically useful for real-world applications. For example, the donut-microVRP have several advantages over the spherical particles such as a larger surface area per volume, a shorter diffusion pathway within and sometimes a better deformability. These unique advantages combined with the tunable chemical composition make the donut-microVRP an interesting class of material for bioencapsulation applications.

As a first proof-of-concept, we employed the nanoclay hydrogel donut-microVRP to encapsulate DNA molecules (Fig. 5a) for improved cell-free protein production. Our previous work shows that the bulk nanoclay hydrogel protects DNA from DNase and hence enhance the cell-free protein production yield $^{30}$. Compared with bulk nanoclay hydrogel, the donut-microVRP have a much higher surface area for mass transfer and are therefore ideal for protein production applications. Here, using a green fluorescent protein (GFP) as a reporter, the nanoclay donut-microVRP-DNA group produces more than three times of GFP than the conventional solutionphase system (Fig. 5b,c). 
a

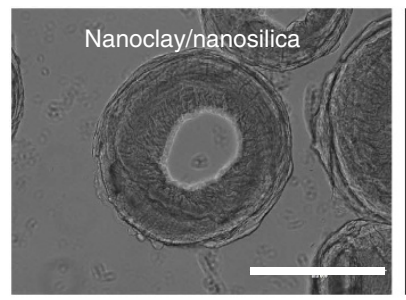

C

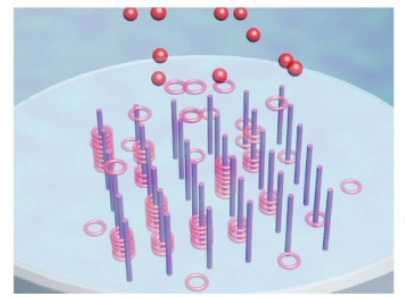

e

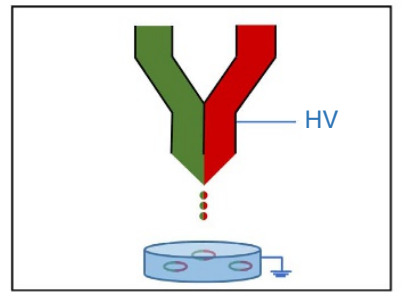

g

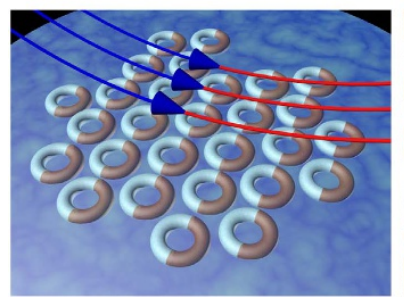

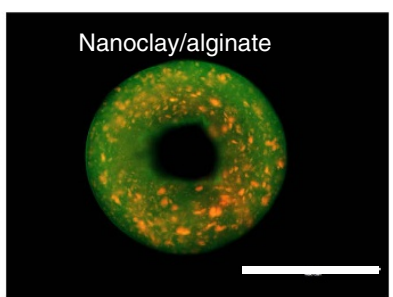
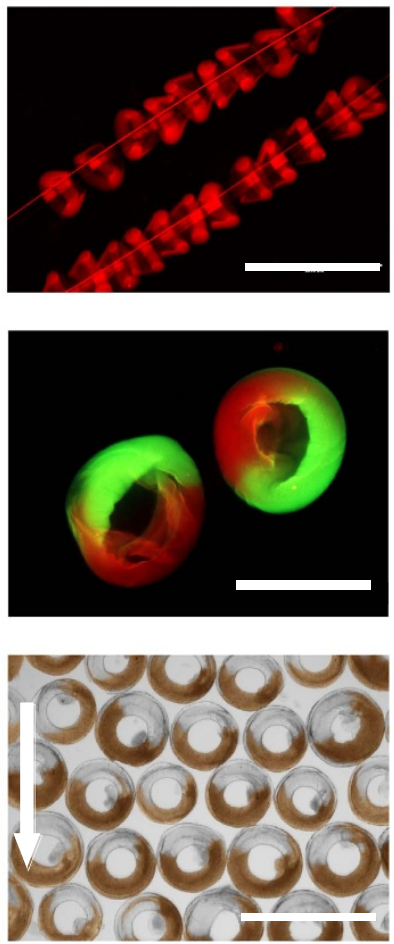

b

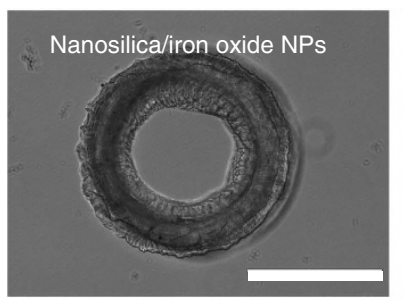

d
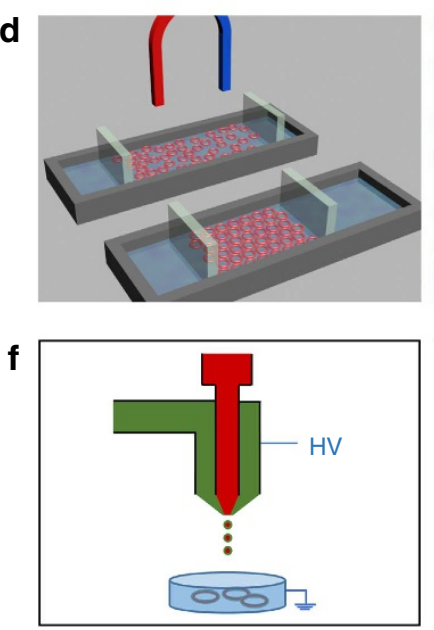
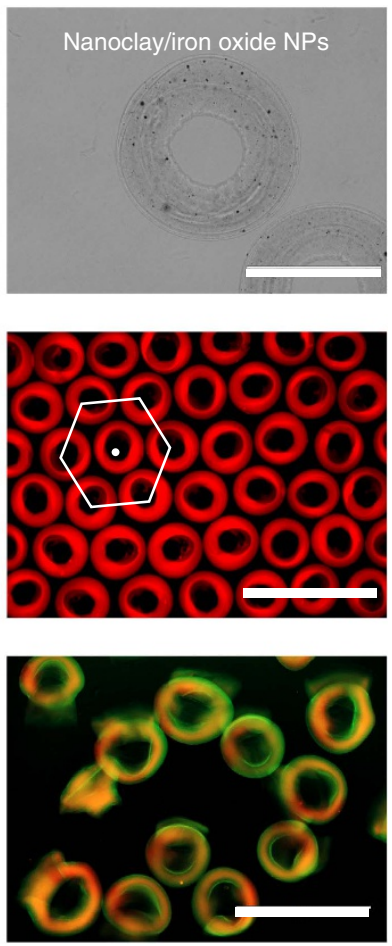

Figure 6 | Composite and assembly of donut-microVRP. (a) Donut-microVRP made from composite materials: nanoclay/nanosilica, nanoclay/alginate. (b) Magnetic donut-microVRP: nanosilica/ $\mathrm{Fe}_{3} \mathrm{O}_{4}$ nanoparticles and nanoclay/ $\mathrm{Fe}_{3} \mathrm{O}_{4}$ nanoparticles. (c) Schematic illustration of the assembly process and a microscopic image of the 1D structure made from nanoclay hydrogel donut-microVRP. (d) Schematic illustration of the assembly process and a microscopic image of the 2D structure made from magnetic nanoclay hydrogel donut-microVRP. (e) Schematic illustration of the nozzle design and a microscopic image of the Janus donut-microVRP. (red: alginate labelled with Alexa Fluor 594 dye; green: alginate labelled with Alexa Fluor 488 dye) (f) Schematic illustration of the nozzle design and a microscopic image of the core-shell donut-microVRP. (g) Directional assembly of the Janus magnetic nanosilica donutmicroVRP; the arrow indicating the direction of the magnetic field. Scale bars, $400 \mu \mathrm{m}(\mathbf{a}, \mathbf{b}) ; 2 \mathrm{~mm}(\mathbf{c}, \mathbf{d}, \mathbf{f}) ; 1 \mathrm{~mm}(\mathbf{e}, \mathbf{g})$.

Next, we demonstrate efficient encapsulation of cells (both prokaryotes and eukaryotes) in hydrogel donut-microVRP. GFPexpressing Escherichia coli cells were encapsulated in the nanoclay hydrogel donut-microVRP (Fig. 5d) and interestingly, the encapsulation did not affect the proliferation of the E. coli (Supplementary Fig. 14) and bacterial donut-microVRP were obtained. Additionally, we encapsulated mammalian cells or cell aggregates in alginate hydrogel donut-VRP (Fig. 5e). Alginate hydrogel has been widely used for cell encapsulation for various applications such as type 1 diabetes treatment ${ }^{38-40}$. The hydrogel provides an immuno-isolated environment for cells while allowing nutrients, oxygen and therapeutics to freely diffuse in and out. For decades, almost all previous works have used spherical alginate particles, typically with a diameter of 500$600 \mu \mathrm{m}$. However, recent studies have shown that it is actually more desirable to use larger size particles (about $1.5 \mathrm{~mm}$ or larger in diameter) for cell delivery because these larger particles generate less foreign body responses upon implantation ${ }^{41}$. Unfortunately, for the large, spherical particles, mass transfer becomes a real problem (for example, the cells in the centre of the spheres can experience the lack of nutrients and oxygen). The non-spherical, donut-VRP, on the other hand, are promising candidates to replace the spherical particles due to their larger surface area and shorter diffusion pathway within (that is, decoupling of the overall dimension and the diffusion distance). To prove the concept, we encapsulated MDA-MB-231 cells, a human breast cancer cell line, in both alginate hydrogel spherical particles and donut-VRP. After four days of culture, the cells in the centre of the spherical particles started to die, while the cells in the donut-VRP remained mostly alive (Supplementary Fig. 15; Fig. 5f). Encouraged by this result, we further demonstrate the feasibility of encapsulating rat pancreatic islets in the alginate hydrogel donut-VRP. The encapsulated islets again showed promising viability after 2 days in vitro culture (Fig. $5 \mathrm{~g}$ ).

Composite and assembly of donut-microVRP. Composite materials often possess unique properties due to synergistic combination of the characteristics of the components. They have been widely used in applications such as catalysis, sensing, actuation, computation and communications $\mathrm{s}^{42}$. To further enhance and diversify the properties and functionalities of the donut-microVRP, we applied the approach of 'freezing' to make VRP with even more complicated compositions and structures. 
First, we demonstrate two different kinds of donut-microVRP made from composite materials: nanoclay/nanosilica and nanoclay/alginate (Fig. 6a). Addition of nanosilica or alginate improves the mechanical strength of the microVRP while maintaining the special characteristics of the nanoclay hydrogel such as enhancement of cell-free protein production. This combination can potentially make the nanoclay/nanosilica donut-microVRP applicable for packing a packed bed reactor for continuous cell-free protein production ${ }^{43,44}$. In addition, we also doped other materials into the current donut-microVRP to introduce new properties. For examples, $\mathrm{Fe}_{3} \mathrm{O}_{4}$ nanoparticles were doped in the nanosilica or nanoclay matrix to prepare magnetic donut-microVRP (Fig. 6b), which can be manipulated using an external magnetic field and potentially used as building blocks for novel stimuli-response materials ${ }^{45}$.

Next, by taking advantage of the unique donut-shape, we directionally assembled the donut-microVRP in either one or two dimensions into supra-structures. For one-dimensional (1D) assembly, we made use of the holes of the donut-microVRP. As shown in the scheme in Fig. 6c, several Nylon short strings were fixed perpendicular to the air-solution interface. When the donutmicroVRP formed, they were threaded into Nylon strings and captured in situ into 1D assemblies (Fig. 6c). For twodimensional assembly, we assembled the magnetic donutmicroVRP into a close-packed monolayer with hexagonal packing symmetry by a combination of magnetic field and a monolayer assembly strategy similar to that used in making Langmuir-Blodgett films (Fig. 6d).

Moreover, by engineering the nozzle used in electrospraying, we fabricated donut-microVRP with compartmentalized structures. As shown in Fig. 6e, the Janus alginate hydrogel donutmicroVRP are produced by using a side-by-side nozzle. Coreshell alginate hydrogel donut-microVRP are also successfully prepared by using a core-shell nozzle (Fig. 6f). These bicompartmental donut-microVRP may find a wide range of applications in biomedical applications, such as co-culture and co-delivery of different types of cells. Additionally, we show another example of magnetic field-assisted directional assembly of Janus donut-microVRP. Janus donut-microVRP with half nanosilica and half nanosilica/ $\mathrm{Fe}_{3} \mathrm{O}_{4}$ nanoparticles are prepared using a side-by-side nozzle. They are aligned under an external magnetic field. Due to their asymmetric magnetic property, all the donut-microVRP are oriented in the same direction with the magnetic field (Fig. 6g).

\section{Discussion}

Compared with spherical particles, non-spherical particles have received much less attention in materials community. However, non-spherical particles such as the donut-shaped ones are often advantageous due to their larger surface areas and shorter diffusion distances. Therefore, mass production of non-spherical particles with controlled dimensions and geometries from different materials will have important implications in fields ranging from catalysis to therapeutic delivery. Here, we report, for the first time, the creation of a new class of non-spherical particles, vortex ring-derived particles. By combining the ubiquitous vortex ring phenomenon with rationally designed freezing events, we develop a universal, robust and scalable method to produce uniform VRP from a variety of materials. Mechanistic studies reveal two critical timescales that determined the VRP shape: the timescale of the vortex ring development and the timescale of the gelation or precipitation process. In order for the VRP to form, the solution properties and process parameters must be well tuned such that the fast evolving vortex rings can be frozen at different stages. Among the various VRP, the donut-
VRP are of particular interest and their diverse applications in bioencapsulation and cell-free protein production are demonstrated. Further engineering of donut-VRP to include compartmentalization and directional assembling are all achieved, creating opportunities to design and engineer VRP-based novel materials that have not been attainable using conventional methods.

\section{Methods}

Materials. All chemicals were used as received. Nanoclay powders (Laponite XLG) were received from Southern Clay Products. Sodium alginate (PRONOVA UP VLVG) was purchased from FMC Corporation. Colloidal silica (LUDOX TM HS-40), chitosan (low molecular weight), Sodium tripolyphosphate, $\mathrm{CaCl}_{2}, \mathrm{MgCl}_{2}$ and acetic acid were purchased from Sigma-Aldrich. $\mathrm{NaH}_{2} \mathrm{PO}_{4}$ was purchased from J.T. Baker.

Preparation of VRP and microVRP. For nanoclay hydrogel VRP, typically, a droplet of $2 \%(\mathrm{w} / \mathrm{v})$ nanoclay solution was extruded from a syringe needle. The droplet fell from a certain height into a $100 \mathrm{mM} \mathrm{CaCl}_{2}$ buffer. The viscosity of the nanoclay solution was tuned by changing the $\mathrm{CaCl}_{2}$ concentration in the nanoclay solution. For nanoclay hydrogel donut-microVRP, a $2 \%(\mathrm{w} / \mathrm{v})$ nanoclay solution was electrosprayed into the $100 \mathrm{mM} \mathrm{CaCl}_{2}$ solution at a voltage of $7.3 \mathrm{kV}$ with a pumping rate of $0.06 \mathrm{ml} \mathrm{min}{ }^{-1}$. The working distance between the nozzle and collecting buffer surface was fixed at $4 \mathrm{~cm}$. For typical alginate hydrogel donutmicroVRP, $2 \%(\mathrm{w} / \mathrm{v})$ sodium alginate solution was electrosprayed into an aqueous buffer of $5 \mathrm{mM} \mathrm{CaCl}$ and $95 \mathrm{mM} \mathrm{MgCl}_{2}$ at $4.7 \mathrm{kV}$ with a pumping rate of $0.06 \mathrm{ml} \mathrm{min}^{-1}$. For typical chitosan hydrogel donut-microVRP, $0.5 \%(\mathrm{w} / \mathrm{v})$ chitosan/acetic acid solution was electrosprayed into acetic acid buffer containing $13.6 \mathrm{mM}$ sodium tripolyphosphate and $50 \mathrm{mM} \mathrm{KH}_{2} \mathrm{PO}_{4}$. Voltage was fixed at $4.8 \mathrm{kV}$ and the pumping rate was $0.3 \mathrm{ml} \mathrm{min}^{-1}$. For nanosilica donut-microVRP, silica nanocolloidal solution was directly electrosprayed into a $100 \mathrm{mM} \mathrm{CaCl}_{2}$ aqueous buffer at $4.6 \mathrm{kV}$ with a pumping rate of $0.06 \mathrm{ml} \mathrm{min}^{-1}$. Other microVRP shapes were obtained by tuning the nanoclay/alginate/chitosan/silica nanocolloidal solution viscosity or electrospraying voltage.

Characterizations. The samples were characterized by different analytical techniques. Scanning electron microscopy (SEM) and EDS element mapping were performed by using a field emission scanning electron micro-analyzer (LEO 1550). Optical and fluorescent microscopic images were observed by a digital inverted microscope (EVOS fl). Confocal laser scanning microscopy (CLSM) images were taken by confocal laser scanning microscope (ZEISS LSM710). High-speed camera images were captured by a high-speed camera (RedLake HG-XL, Integrated Design Tools).

Cell/bacteria encapsulation. For cell/bacteria encapsulation, MDA-MB-231 cells, isolated rat pancreatic islets or E. coli were collected by centrifugation, after removing the supernatant, cells or cell aggregates were re-suspended into a $2 \%$ sodium alginate solution. This cell/alginate solution was electrosprayed into the $95 \mathrm{mM} \mathrm{MgCl}_{2} / 5 \mathrm{mM} \mathrm{CaCl}_{2}$ buffer. Then, the donut-microVRP were collected and washed by PBS for three times to remove excess crosslinking buffer and transferred into corresponding culture medium.

Cell-free production of model protein. To produce green fluorescent protein (GFP), $1.4 \mu \mathrm{g}$ of expression plasmid (PIVEX 2.4 containing GFP (5PRIME)) was added to a solution containing $5 \mathrm{mg}$ of nanoclay hydrogel donut-microVRP, $37.8 \mu \mathrm{l}$ of cell-free lysate, and $15 \mu \mathrm{l}$ of nuclease free water. Samples were then placed in a BioTek fluorescent plate reader and measured every $30 \mathrm{~min}$ with an excitation of $488 \mathrm{~nm}$ and an emission of $509 \mathrm{~nm}$. In order to determine the effect of nanoclay hydrogel donut-microVRP on protein expression, samples were done in triplicate and side-by-side with control samples. All samples were then imaged at the 24 -h time point.

Data availability. The data that support the findings of this study are available from the corresponding author upon request.

\section{References}

1. Birch, J. M. \& Dickinson, M. H. Spanwise flow and the attachment of the leading-edge vortex on insect wings. Nature 412, 729-733 (2001).

2. Hu, D. L., Chan, B. \& Bush, J. W. M. The hydrodynamics of water strider locomotion. Nature 424, 663-666 (2003).

3. Chakraborty, P., Gioia, G. \& Kieffer, S. W. Volcanic mesocyclones. Nature 458, 497-500 (2009).

4. Lee, J. S. et al. Origin and dynamics of vortex rings in drop splashing. Nat. Commun. 6, 8187 (2015). 
5. Thomson, J. \& Newall, H. On the formation of vortex rings by drops falling into liquids, and some allied phenomena. Proc. R. Soc. Lond. 39, 417-436 (1885).

6. Hsiao, M. Y., Lichter, S. \& Quintero, L. G. The critical weber number for vortex and jet formation for drops impinging on a liquid pool. Phys. Fluids 31, 3560-3562 (1988).

7. Lim, T. T. \& Nickels, T. B. Instability and reconnection in the head-on collision of 2 vortex rings. Nature 357, 225-227 (1992)

8. Peck, B. \& Sigurdson, L. The 3-dimensional vortex structure of an impacting water drop. Phys. Fluids 6, 564-576 (1994).

9. Cresswell, R. W. \& Morton, B. R. Drop-formed vortex rings-the generation of vorticity. Phys. Fluids 7, 1363-1370 (1995).

10. Dooley, B. S., Warncke, A. E., Gharib, M. \& Tryggvason, G. Vortex ring generation due to the coalescence of a water drop at a free surface. Exp. Fluids 22, 369-374 (1997).

11. Zou, J. et al. Large bubble entrapment during drop impacts on a restricted liquid surface. Phys. Fluids 24, 7 (2012).

12. Kleckner, D. \& Irvine, W. T. M. Creation and dynamics of knotted vortices. Nat. Phys 9, 253-258 (2013).

13. Walker, T. W., Logia, A. N. \& Fuller, G. G. Multiphase flow of miscible liquids: jets and drops. Exp. Fluids 56, 14 (2015).

14. Agbaglah, G. et al. Drop impact into a deep pool: vortex shedding and jet formation. J. Fluid Mech. 764, 12 (2015).

15. Wilga, C. D. \& Lauder, G. V. Biomechanics-hydrodynamic function of the shark's tail. Nature 430, 850-850 (2004).

16. Linden, P. F. \& Turner, J. S. 'Optimal' vortex rings and aquatic propulsion mechanisms. Proc. R. Soc. B-Biol. Sci. 271, 647-653 (2004).

17. Hove, J. R. et al. Intracardiac fluid forces are an essential epigenetic factor for embryonic cardiogenesis. Nature 421, 172-177 (2003).

18. Dabiri, J. O. \& Gharib, M. Fluid entrainment by isolated vortex rings. J. Fluid Mech. 511, 311-331 (2004).

19. Gharib, M., Rambod, E., Kheradvar, A., Sahn, D. J. \& Dabiri, J. O. Optimal vortex formation as an index of cardiac health. Proc. Natl Acad. Sci. USA 103, 6305-6308 (2006).

20. O’Farrell, C. \& Dabiri, J. O. Nested contour dynamics models for axisymmetric vortex rings and vortex wakes. J. Fluid Mech. 748, 521-548 (2014)

21. Ruiz, L. A., Whittlesey, R. W. \& Dabiri, J. O. Vortex-enhanced propulsion. J. Fluid Mech. 668, 5-32 (2011).

22. Chang, Y. K. \& Vakili, A. D. Dynamics of vortex rings in cross-flow. Phys Fluids 7, 1583-1597 (1995).

23. Ungphaiboon, S. et al. Materials for microencapsulation: what toroidal particles ('doughnuts') can do better than spherical beads. Soft Matter 6, 4070-4083 (2010).

24. Sharma, V., Szymusiak, M., Shen, H., Nitsche, L. C. \& Liu, Y. Formation of polymeric toroidal-spiral particles. Langmuir 28, 729-735 (2012).

25. Szymusiak, M., Sharma, V., Nitsche, L. C. \& Liu, Y. Interaction of sedimenting drops in a miscible solution-formation of heterogeneous toroidal-spiral particles. Soft Matter 8, 7556-7559 (2012).

26. Hu, P. et al. Ion-induced synthesis of uniform single-crystalline sulphide-based quaternary-alloy hexagonal nanorings for highly efficient photocatalytic hydrogen evolution. Adv. Mater. 25, 2567-2572 (2013).

27. Du, Y. N. et al. Sequential assembly of cell-laden hydrogel constructs to engineer vascular-like microchannels. Biotechnol. Bioeng. 108, 1693-1703 (2011).

28. Shariff, K. \& Leonard, A. Vortex rings. Annu. Rev. Fluid Mech. 24, U235-U279 (1992)

29. Ruzicka, B. et al. Observation of empty liquids and equilibrium gels in a colloidal clay. Nat. Mater. 10, 56-60 (2011).

30. Yang, D. Y. et al. Enhanced transcription and translation in clay hydrogel and implications for early life evolution. Sci. Rep. 3, 3165 (2013).

31. Dendukuri, D., Pregibon, D. C., Collins, J., Hatton, T. A. \& Doyle, P. S. Continuous-flow lithography for high-throughput microparticle synthesis. Nat. Mater. 5, 365-369 (2006)

32. Fang, A., Gaillard, C. \& Douliez, J.-P. Template-free formation of monodisperse doughnut-shaped silica microparticles by droplet-based microfluidics. Chem. Mater. 23, 4660-4662 (2011).

33. Tian, Y., Wu, G., Tian, X., Tao, X. \& Chen, W. Novel erythrocyte-like graphene microspheres with high quality and mass production capability via electrospray assisted self-assembly. Sci. Rep. 3, 3327 (2013).
34. Xie, J. W., Jiang, J., Davoodi, P., Srinivasan, M. P. \& Wang, C. H. Electrohydrodynamic atomization: a two-decade effort to produce and process micro-/nanoparticulate materials. Chem. Eng. Sci. 125, 32-57 (2015).

35. Sridhar, R. et al. Electrosprayed nanoparticles and electrospun nanofibers based on natural materials: applications in tissue regeneration, drug delivery and pharmaceuticals. Chem. Soc. Rev. 44, 790-814 (2015).

36. Lu, Y. C. et al. Designing compartmentalized hydrogel microparticles for cell encapsulation and scalable 3D cell culture. J. Mat. Chem. B 3, 353-360 (2015)

37. Wang, X. X. et al. Monodisperse erythrocyte-sized and acid-soluble chitosan microspheres prepared via electrospraying. RSC Adv. 5, 34243-34250 (2015).

38. Soonshiong, P. et al. Insulin independence in a type- 1 diabetic patient after encapsulated islet transplantation. Lancet 343, 950-951 (1994).

39. An, D. et al. Developing robust, hydrogel-based, nanofiber-enabled encapsulation devices (NEEDs) for cell therapies. Biomaterials 37, 40-48 (2015).

40. Ma, M. et al. Core-shell hydrogel microcapsules for improved islets encapsulation. Adv. Healthc. Mater. 2, 667-672 (2013).

41. Veiseh, O. et al. Size- and shape-dependent foreign body immune response to materials implanted in rodents and non-human primates. Nat. Mater. 14, 643-U125 (2015)

42. McEvoy, M. A. \& Correll, N. Materials that couple sensing, actuation, computation, and communication. Science 347, 1261689 (2015).

43. Chu, L. \& Robinson, D. K. Industrial choices for protein production by large-scale cell culture. Curr. Opin. Biotechnol. 12, 180-187 (2001).

44. Park, N., Um, S. H., Funabashi, H., Xu, J. F. \& Luo, D. A cell-free protein-producing gel. Nat. Mater. 8, 432-437 (2009).

45. Fusco, S. et al. An integrated microrobotic platform for on-demand, targeted therapeutic interventions. Adv. Mater. 26, 952-957 (2014).

\section{Acknowledgements}

We thank J. An, W. Song, Y. Li, T. Liu for insightful discussions. The work was partially supported by the American Diabetes Association (7-13-JF-42 to M.M.), the 3M Company (to M.M.), the SUNY Research Foundation (to M.M.), the National Institutes of Health (Grant 1R03EB020910-01 to M.M.) and the National Science Foundation (Award number 1530522 to D.L., M.M. and P.S.). The work took use of the Cornell Center for Materials Research shared facilities that are supported through the NSF MRSEC programme (DMR-1120296).

\section{Author contributions}

D.A., D.L. and M.M. conceived the design of the study. D.A. fabricated the hydrogel and nanosilica VRP. A.W. did the simulation. K.G.Y. did the cell-free protein production experiments. C.-T.C., D.A. conducted the fluid mechanics study. D.A., A.W., K.G.Y., C.-T.C., V.R.K., A.K.D., P.H.S., D.L. and M.M. contributed to the data analysis. D.A., A.W., C.-T.C., P.H.S., D.L. and M.M. wrote the manuscript.

\section{Additional information}

Supplementary Information accompanies this paper at http://www.nature.com/ naturecommunications

Competing financial interests: The authors declare no competing financial interests

Reprints and permission information is available online at http://npg.nature.com/ reprintsandpermissions/

How to cite this article: An, D. et al. Mass production of shaped particles through vortex ring freezing. Nat. Commun. 7:12401 doi: 10.1038/ncomms12401 (2016).

This work is licensed under a Creative Commons Attribution 4.0 International License. The images or other third party material in this article are included in the article's Creative Commons license, unless indicated otherwise in the credit line; if the material is not included under the Creative Commons license, users will need to obtain permission from the license holder to reproduce the material. To view a copy of this license, visit http://creativecommons.org/licenses/by/4.0/

(C) The Author(s) 2016 